\title{
BIFURCATION AND CONTROL FOR A DISCRETE-TIME PREY-PREDATOR MODEL WITH HOLLING-IV FUNCTIONAL RESPONSE
}

\author{
QiAOLING CHEN*, Zhidong TENG *, ZENGYUN HU *,** \\ * College of Mathematics and System Sciences \\ Xinjiang University, Urumqi 830046, People's Republic of China \\ e-mail: zhidong@xju.edu.cn, zhidong_teng@sina.com \\ ** State Key Laboratory of Desert and Oasis Ecology, Xinjiang Institute of Ecology and Geography \\ Chinese Academy of Sciences, Urumqi 830011, People's Republic of China
}

\begin{abstract}
The dynamics of a discrete-time predator-prey model with Holling-IV functional response are investigated. It is shown that the model undergoes a flip bifurcation, a Hopf bifurcation and a saddle-node bifurcation by using the center manifold theorem and bifurcation theory. Numerical simulations not only exhibit our results with the theoretical analysis, but also show the complex dynamical behaviors, such as the period-3, 6, 9, 12, 20,63, 70, 112 orbits, a cascade of period-doubling bifurcations in period-2, 4, 8, 16, quasi-periodic orbits, an attracting invariant circle, an inverse period-doubling bifurcation from the period-32 orbit leading to chaos and a boundary crisis, a sudden onset of chaos and a sudden disappearance of the chaotic dynamics, attracting chaotic sets and non-attracting sets. We also observe that when the prey is in chaotic dynamics the predator can tend to extinction or to a stable equilibrium. Specifically, we stabilize the chaotic orbits at an unstable fixed point by using OGY chaotic control.
\end{abstract}

Keywords: discrete prey-predator model, flip bifurcation, Hopf bifurcation, saddle-node bifurcation, OGY chaotic control.

\section{Introduction}

For the purpose of system modelling and analysis in mathematical biology, discrete-time models are more considered an essential tool. Firstly, discrete-time models are more suitable for describing systems which evolve over time. Secondly, compared with continuous-time models, the advantage they offer is that they are generally more direct, more convenient and more accurate to formulate. Thirdly, recent works have shown that for discrete-time models the dynamics can produce a much richer set of patterns than those observed in continuous-time models (Busłowicz, 2010; Busłowicz and Ruszewski, 2012; Duda, 2012; Feedman, 1980; Holling, 1965; Huang and Xiao, 2004; Hsu, 1978; Raja et al., 2011; Xu et al., 2011; Zhang et al., 2011). At last, we can get more interesting dynamical behaviors and more accurate numerical simulations results from the discrete-time models; moreover, numerical simulations of continuous-time models are obtained by discretizing the models.

Until now, many works have concerned discrete-time two-species predator-prey models (Jing and Yang, 2006;
Guckenheimer and Holmes, 1983; Wiggins, 1990; He and Lai, 2011; Liu and Xiao, 2007; Hu et al., 2011; Tong et al., 2012). In these articles, the main studied topics are the existence and local stability of an equilibrium, flip bifurcation and Hopf bifurcation by using the center manifold theorem and bifurcation theory.

Tong et al. (2012) discussed the existence of periodic solutions for discrete semi-ratio-dependent prey-predator models with functional response. The permanence in a delayed discrete prey-predator model with Holling-III functional response was discussed by Fan and Li (2004). Kuznetsov (1998) summarized local stability conditions for equilibria, and used numerical computation to obtain codim-1 bifurcation curves that emanate at codim-2 bifurcation points in order to compute the stability boundaries of cycles with periods $4,5,8$ and 16 , but without a theorematic proof.

It is well known that continuous-time two-species prey-predator model with Holling-IV functional response is described in the following form (Liu et al., 2010): 


$$
\left\{\begin{array}{c}
\dot{x_{1}}=x_{1}\left(1-x_{1}-k x_{1}^{2}-\frac{x_{2}}{x_{1}^{2}+\beta^{2}}\right), \\
\dot{x_{2}}=x_{2}\left(-\delta_{0}-\delta_{1} x_{2}+\frac{\gamma x_{1}}{x_{1}^{2}+\beta^{2}}\right)
\end{array}\right.
$$

where $x_{1}(t)$ and $x_{2}(t)$ represent prey and predator densities, respectively, $k, \beta, \delta_{1}, \gamma$ are positive constants, and $\delta_{0}$ is a constant. Applying the Euler forward scheme to model (1), we obtain the following discrete-time model:

$$
\left\{\begin{array}{c}
x_{1}(n+1) \\
=x_{1}(n)+\delta x_{1}(n)\left(1-x_{1}(n)\right. \\
\left.\quad-k x_{1}^{2}(n)-\frac{x_{2}(n)}{x_{1}^{2}(n)+\beta^{2}}\right), \\
x_{2}(n+1) \\
=x_{2}(n)+\delta x_{2}(n)\left(-\delta_{0}\right. \\
\left.\quad-\delta_{1} x_{2}(n)+\frac{\gamma x_{1}(n)}{x_{1}^{2}(n)+\beta^{2}}\right),
\end{array}\right.
$$

where $\delta>0$ is the time step size. In this paper, we investigate the bifurcation problems of the model (2) in the interior of the first quadrant $\mathbb{R}_{+}^{2}$ by using bifurcation theory and the center manifold theorem. We will prove that the model (2) possesses flip bifurcation, Hopf bifurcation and saddle-node bifurcation. Furthermore, by using the OGY method, we will study the chaotic control of the model (2).

This paper is organized as follows. In Section 2, we study the existence and local stability of equilibria of the model (2). In Sections 3-5, the sufficient conditions on the existence of flip bifurcation, Hopf bifurcation and saddle-node bifurcation are established. In Section 6, the OGY controller to eliminate the unstable periodic orbits is designed. In Section 7, numerical simulations are given to illustrate the results obtained in this paper. In Section 8, we provide a discussion to conclude this paper.

\section{Local stability of equilibria}

From the point of view of biology, we will focus on the dynamical behaviors of the model (2) in the closed first quadrant $\mathbb{R}_{+}^{2}$ of the $\left(x_{1}, x_{2}\right)$ plane. It is clear that the equilibria of (2) satisfy the following equations:

$$
\left\{\begin{array}{l}
x_{1}=x_{1}+\delta x_{1}\left(1-x_{1}-k x_{1}^{2}-\frac{x_{2}}{x_{1}^{2}+\beta^{2}}\right), \\
x_{2}=x_{2}+\delta x_{2}\left(-\delta_{0}-\delta_{1} x_{2}+\frac{\gamma x_{1}}{x_{1}^{2}+\beta^{2}}\right) .
\end{array}\right.
$$

We have the following results.

\section{Lemma 1.}

(i) The model (2) always has two equilibria, $E_{0}(0,0)$ and $E_{1}(m, 0)$, where $m=\frac{-1+\sqrt{1+4 k}}{2 k}$. (ii) If $\delta_{0} \geq 0$ and $\delta_{0}\left(m^{2}+\beta^{2}\right)<\gamma m$ or $\delta_{0}<0$ and $\delta_{1} \beta^{2}>-\delta_{0}$, then the model (2) also has at least a positive equilibrium $E_{2}\left(x_{1}^{*}, x_{2}^{*}\right)$, where $\left(x_{1}^{*}, x_{2}^{*}\right)$ satisfies the following equations:

$$
\left\{\begin{array}{l}
0=1-x_{1}-k x_{1}^{2}-\frac{x_{2}}{x_{1}^{2}+\beta^{2}}, \\
0=-\delta_{0}-\delta_{1} x_{2}+\frac{\gamma x_{1}}{x_{1}^{2}+\beta^{2}}, \quad 0<x_{1}<m .
\end{array}\right.
$$

Proof. The conclusion (i) is obvious. Let

$$
\begin{aligned}
I\left(x_{1}\right)= & \delta_{1}\left(1-x_{1}-k x_{1}^{2}\right)\left(x_{1}^{2}+\beta^{2}\right)^{2} \\
& +\delta_{0}\left(x_{1}^{2}+\beta^{2}\right)-\gamma x_{1} .
\end{aligned}
$$

When the conditions of Lemma 1 hold, we have $I(0)=$ $\delta_{0} \beta^{2}+\delta_{1} \beta^{4}>0$ and $I(m)=\delta_{0}\left(m^{2}+\beta^{2}\right)-\gamma m<0$. Hence, there exists at least a positive $x_{1}^{*} \in(0, m)$ such that $I\left(x_{1}^{*}\right)=0$. Let $x_{2}^{*}=\frac{1}{\delta_{1}}\left(-\delta_{0}+\frac{\gamma x_{1}^{*}}{x_{1}^{* 2}+\beta^{2}}\right)$. Then $x_{2}^{*}>0$. Obviously, $\left(x_{1}^{*}, x_{2}^{*}\right)$ satisfies Eqn. (4). Therefore, the conclusion (ii) is proved. This completes the proof.

Now, we study the stability of these equilibria of model (2) by using a linearization method and a Jacobian matrix.

A Jacobian matrix at $E_{0}(0,0)$ is

$$
J\left(E_{0}\right)=\left(\begin{array}{cc}
1+\delta & 0 \\
0 & 1-\delta \delta_{0}
\end{array}\right) .
$$

Two eigenvalues of $J\left(E_{0}\right)$ are $\lambda_{1}=1+\delta$ and $\lambda_{2}=1-$ $\delta \delta_{0}$. We see that $E_{0}(0,0)$ is always unstable, and $E_{0}(0,0)$ is non-hyperbolic if $\delta=2 / \delta_{0}$.

We can see that when $\delta=2 / \delta_{0}$, one of the eigenvalues of $J\left(E_{0}\right)$ is -1 , and the other is neither 1 nor -1 . Thus, flip bifurcation may occur when parameters vary in a neighborhood of $\delta=2 / \delta_{0}$.

A Jacobian matrix at $E_{1}(m, 0)$ is

$$
\begin{aligned}
& J\left(E_{1}\right) \\
& =\left(\begin{array}{cc}
1+\delta\left(-m-2 k m^{2}\right) & -\frac{\delta m}{m^{2}+\beta^{2}} \\
0 & 1+\delta\left(-\delta_{0}+\frac{\gamma m}{m^{2}+\beta^{2}}\right)
\end{array}\right) .
\end{aligned}
$$

Two eigenvalues of $J\left(E_{1}\right)$ are

$$
\lambda_{1}=1+\delta\left(-m-2 k m^{2}\right)
$$

and

$$
\lambda_{2}=1+\delta\left(-\delta_{0}+\frac{\gamma m}{m^{2}+\beta^{2}}\right) .
$$


Obviously, $E_{1}(m, 0)$ is asymptotically stable if and only if

$$
\delta_{0}>\frac{\gamma m}{m^{2}+\beta^{2}}
$$

and

$$
\delta<\min \left\{\frac{2}{m+2 k m^{2}}, \frac{2\left(m^{2}+\beta^{2}\right)}{\delta_{0}\left(m^{2}+\beta^{2}\right)-\gamma m}\right\} .
$$

$E_{1}(m, 0)$ is unstable if and only if

$$
\delta_{0}<\frac{\gamma m}{m^{2}+\beta^{2}}
$$

or

$$
\delta>\min \left\{\frac{2}{m+2 k m^{2}}, \frac{2\left(m^{2}+\beta^{2}\right)}{\delta_{0}\left(m^{2}+\beta^{2}\right)-\gamma m}\right\},
$$

and $E_{1}(m, 0)$ is non-hyperbolic if and only if

$$
\delta=\frac{2}{m+2 k m^{2}}
$$

or

$$
\delta_{0}=\frac{\gamma m}{m^{2}+\beta^{2}}
$$

or

$$
\delta_{0}>\frac{\gamma m}{m^{2}+\beta^{2}}, \quad \delta=\frac{2\left(m^{2}+\beta^{2}\right)}{\delta_{0}\left(m^{2}+\beta^{2}\right)-\gamma m} .
$$

We can see that one of the eigenvalues of $J\left(E_{1}\right)$ is -1 and the other is neither 1 nor -1 when the parameters of the model (2) are located in one of the following sets:

$$
\begin{aligned}
F_{A}= & \left\{\left(\delta_{1}, k, \beta, \delta_{0}, \gamma, \delta\right): \delta=\frac{2}{m+2 k m^{2}},\right. \\
& \left.\delta_{0} \neq \frac{\gamma m}{m^{2}+\beta^{2}}, \delta \neq \frac{2 m^{2}+2 \beta^{2}}{\delta_{0}\left(m^{2}+\beta^{2}\right)-\gamma m}\right\} .
\end{aligned}
$$

$E_{1}(m, 0)$ can undergo flip bifurcation when all parameters of the model (2) vary in a small neighborhood of $F_{A}$. When the parameters are in $F_{A}$, a center manifold of the model (2) is $x_{2}=0$, and (2) restricted to this center manifold is

$$
x_{1}(n+1)=x_{1}(n)+\delta x_{1}(n)\left(1-x_{1}(n)-k x_{1}^{2}(n)\right) .
$$

This shows that the predator becomes extinction and the prey undergoes flip bifurcation to chaos on choosing bifurcation parameter $\delta$.

In Section 7, we will give an example to show that flip bifurcation at $E_{1}(m, 0)$ occurs when the parameters are in $F_{A}$ with bifurcation parameter $\delta$.
A Jacobian matrix at $E_{2}\left(x_{1}^{*}, x_{2}^{*}\right)$ is

$$
\begin{aligned}
J\left(E_{2}\right)=\left(\begin{array}{c}
1+\delta\left(-x_{1}^{*}-2 k x_{1}^{* 2}+\frac{2 x_{1}^{* 2} x_{2}^{*}}{\left(x_{1}^{* 2}+\beta^{2}\right)^{2}}\right) \\
\frac{\gamma \delta x_{2}^{*}\left(\beta^{2}-x_{1}^{* 2}\right)}{\left(x_{1}^{* 2}+\beta^{2}\right)^{2}} \\
\\
\\
\quad \frac{-\delta x_{1}^{*}}{x_{1}^{* 2}+\beta^{2}} \\
1-\delta \delta_{1} x_{2}^{*}
\end{array}\right) .
\end{aligned}
$$

The characteristic equation of $J\left(E_{2}\right)$ can be written as

$$
\lambda^{2}+p\left(E_{2}\right) \lambda+q\left(E_{2}\right)=0,
$$

where

$$
p\left(E_{2}\right)=-2-G \delta, \quad q\left(E_{2}\right)=1+G \delta+H \delta^{2},
$$

with

$$
G=-x_{1}^{*}-2 k x_{1}^{* 2}-\delta_{1} x_{2}^{*}+\frac{2 x_{1}^{* 2} x_{2}^{*}}{\left(x_{1}^{* 2}+\beta^{2}\right)^{2}},
$$

and

$$
\begin{aligned}
H= & \delta_{1} x_{1}^{*} x_{2}^{*}+2 k \delta_{1} x_{1}^{* 2} x_{2}^{*} \\
& +\frac{\gamma x_{1}^{*} x_{2}^{*}\left(\beta^{2}-x_{1}^{* 2}\right)}{\left(x_{1}^{* 2}+\beta^{2}\right)^{3}}-\frac{2 \delta_{1} x_{1}^{* 2} x_{2}^{* 2}}{\left(x_{1}^{* 2}+\beta^{2}\right)^{2}} .
\end{aligned}
$$

Two eigenvalues of $J\left(E_{2}\right)$ are

$$
\lambda_{1,2}=1+\frac{1}{2} \delta\left(G \pm \sqrt{G^{2}-4 H}\right) .
$$

\section{Proposition 1.}

(i) $E_{2}\left(x_{1}^{*}, x_{2}^{*}\right)$ is asymptotically stable if one of the following conditions holds:

$$
\begin{aligned}
& \text { (i.l) }-2 \sqrt{H} \leq G<0, H>0 \text {, and } \delta<-\frac{G}{H} . \\
& \text { (i.2) } G<-2 \sqrt{H}, H>0 \text {, and } \delta<-\frac{G+\sqrt{G^{2}-4 H}}{H} \text {. }
\end{aligned}
$$

(ii) $E_{2}\left(x_{1}^{*}, x_{2}^{*}\right)$ is unstable if one of the following conditions holds:

$$
\begin{aligned}
& \text { (ii.1) }-2 \sqrt{H} \leq G<0, H>0 \text {, and } \delta>-\frac{G}{H} . \\
& \text { (ii.2) } G<-2 \sqrt{H}, H>0 \text {, and } \delta>-\frac{G+\sqrt{G^{2}-4 H}}{H} \text {. }
\end{aligned}
$$

(iii) $E_{2}\left(x_{1}^{*}, x_{2}^{*}\right)$ is non-hyperbolic if one of the following conditions holds:

(iii.1) $-2 \sqrt{H} \leq G<0, H>0$, and $\delta=-\frac{G}{H}$.

(iii.2) $G<-2 \sqrt{H}, H>0, \delta=-\frac{G+\sqrt{G^{2}-4 H}}{H}$ or $\delta=-\frac{G-\sqrt{G^{2}-4 H}}{H}$.

(iii.3) $H=0$, which is equivalent to

$$
\delta_{1}=\frac{D}{\left(x_{1}^{* 2}+\beta^{2}\right)^{3}\left(1+2 k x_{1}^{*}\right)},
$$

where

$$
D=\gamma\left(3 x_{1}^{* 2}-\beta^{2}\right)-2 \delta_{0} x_{1}^{*}\left(x_{1}^{* 2}+\beta^{2}\right) .
$$


We easily see that one of the eigenvalues of $J\left(E_{2}\right)$ is -1 and the other is neither 1 nor -1 when the parameters of the model (2) are located in the following sets:

$$
\begin{gathered}
F_{31}=\left\{\left(\delta_{1}, k, \beta, \delta_{0}, \gamma, \delta\right): G<-2 \sqrt{H},\right. \\
\delta=-\frac{G+\sqrt{G^{2}-4 H}}{H}, H>0, \\
\left.\delta \neq-\frac{2}{G}, \delta \neq-\frac{4}{G}, \delta_{0}>0\right\},
\end{gathered}
$$

and

$$
\begin{gathered}
F_{32}=\left\{\left(\delta_{1}, k, \beta, \delta_{0}, \gamma, \delta\right): G<-2 \sqrt{H},\right. \\
\delta=-\frac{G-\sqrt{G^{2}-4 H}}{H}, H>0, \\
\left.\delta \neq-\frac{2}{G}, \delta \neq-\frac{4}{G}, \delta_{0}>0\right\} .
\end{gathered}
$$

We can obtain that the eigenvalues of $J\left(E_{2}\right)$ are a pair of conjugate complex numbers with module 1 when the parameters of the model (2) are located in the following set:

$$
\begin{aligned}
F_{33}= & \left\{\left(\delta_{1}, k, \beta, \delta_{0}, \gamma, \delta\right):-2 \sqrt{H} \leq G<0,\right. \\
& \left.H>0, \delta=-\frac{G}{H}, \delta_{0}>0\right\} .
\end{aligned}
$$

We can also obtain that one of the eigenvalues of $J\left(E_{2}\right)$ is 1 and the other is neither 1 nor -1 when the parameters of the model (2) are located in the following set:

$$
\begin{aligned}
F_{34}=\left\{\left(\delta_{1}, k, \beta, \delta_{0}, \gamma, \delta\right):\right. \\
\delta_{1}=\frac{D}{\left(x_{1}^{* 2}+\beta^{2}\right)^{3}\left(1+2 k x_{1}^{*}\right)}, \\
\delta_{1} \neq \frac{2 \gamma x_{1}^{* 2}-2 \delta_{0} x_{1}^{*}\left(x_{1}^{* 2}+\beta^{2}\right)}{\left(x_{1}^{* 2}+\beta^{2}\right) D_{1}}, \\
\delta_{1} \neq \frac{2 \delta x_{1}^{* 2}\left[\gamma x_{1}-\delta_{0}\left(x_{1}^{* 2}+\beta^{2}\right)\right]}{\left(x_{1}^{* 2}+\beta^{2}\right) D_{2}}, \\
\left.\delta_{0}<0\right\} .
\end{aligned}
$$

where

$$
\begin{aligned}
& D_{1}=\gamma \delta x_{1}^{*}+\left(x_{1}^{* 2}+\beta^{2}\right)\left(x_{1}^{*}+2 k x_{1}^{* 2}-\delta \delta_{0}\right), \\
& D_{2}=\gamma \delta x_{1}^{*}+\left(x_{1}^{* 2}+\beta^{2}\right)\left(x_{1}^{*}+2 k x_{1}^{* 2}-\delta \delta_{0}-2\right) .
\end{aligned}
$$

In the following sections, by using the center manifold theorem and bifurcation theory given by Robinson (1999) and Guckenheimer (1983), we will study flip bifurcation of $E_{2}\left(x_{1}^{*}, x_{2}^{*}\right)$ if all parameters of the model (2) vary in a small neighborhood of $F_{31}$ (or $F_{32}$ ), Hopf bifurcation of $E_{2}\left(x_{1}^{*}, x_{2}^{*}\right)$ if all parameters of the model (2) vary in a small neighborhood of $F_{33}$, and saddle-node bifurcation of $E_{2}\left(x_{1}^{*}, x_{2}^{*}\right)$ if all parameters of the model (2) vary in a small neighborhood of $F_{34}$.

In the following discussion, for convenience, for a function $f\left(x_{1}, x_{2}, \ldots, x_{n}\right)$, we shall write $f_{x_{i}}, f_{x_{i} x_{j}}$, and $f_{x_{i} x_{j} x_{k}}$ for the first order, second order, and third order partial derivative of $f\left(x_{1}, x_{2}, x_{3}, \ldots, x_{n}\right)$ with respect to $x_{i}, x_{j}$, and $x_{k}$, respectively.

\section{Flip bifurcation}

In this and the following three sections, we always assume that the model (2) has a positive equilibrium $E_{2}\left(x_{1}^{*}, x_{2}^{*}\right)$, that is, the condition (ii) of Lemma 1 always holds.

We first discuss flip bifurcation of the model (2) at $E_{2}\left(x_{1}^{*}, x_{2}^{*}\right)$ when parameters $\left(\delta_{1}, k, \beta, \delta_{0}, \gamma, \delta\right)$ vary in a small neighborhood of $F_{31}$. Similar arguments can be applied to the case $F_{32}$.

Taking parameters $\left(\delta_{1}, k, \beta, \delta_{0}, \gamma, \delta\right)=$ $\left(\delta_{11}, k_{1}, \beta_{1}, \delta_{01}, \gamma_{1}, \bar{\delta}\right) \in F_{31}$. Further, giving $\delta$ a perturbation $\delta^{*}$ at $\bar{\delta}$, we consider a perturbation of the model (2) as follows:

$$
\left\{\begin{aligned}
x_{1}(n+1) & \\
= & x_{1}(n)+\left(\bar{\delta}+\delta^{*}\right) x_{1}(n)\left(1-x_{1}(n)\right. \\
& \left.-k_{1} x_{1}(n)^{2}-\frac{x_{2}(n)}{x_{1}^{2}(n)+\beta_{1}^{2}}\right) \\
\triangleq & f\left(x_{1}(n), x_{2}(n), x_{3}\right), \\
x_{2} & (n+1) \\
= & x_{2}(n)+\left(\bar{\delta}+\delta^{*}\right) x_{2}(n)\left(-\delta_{01}\right. \\
& \left.-\delta_{11} x_{2}(n)+\frac{\gamma x_{1}(n)}{x_{1}^{2}(n)+\beta^{2}}\right) \\
\triangleq & g\left(x_{1}(n), x_{2}(n), x_{3}\right),
\end{aligned}\right.
$$

where $\left|\delta^{*}\right| \ll 1$, and $x_{3}=\delta^{*}$.

Let $u_{1}(n)=x_{1}(n)-x_{1}^{*}, u_{2}(n)=x_{2}(n)-x_{2}^{*}$, then equilibrium $E_{2}\left(x_{1}^{*}, x_{2}^{*}\right)$ is transformed into the origin, and further expanding $f$ and $g$ as a Taylor series at $\left(u_{1}, u_{2}, x_{3}\right)=(0,0,0)$ to the third order, the model (24) becomes

$$
\left\{\begin{aligned}
u_{1}( & +1) \\
= & a_{1} u_{1}(n)+a_{2} u_{2}(n)+a_{11} u_{1}^{2}(n) \\
& +a_{11} u_{1}(n) u_{2}(n)+a_{13} u_{1}(n) x_{3} \\
& +a_{23} u_{2}(n) x_{3}+a_{111} u_{1}^{3}(n) \\
& +a_{112} u_{1}^{2}(n) u_{2}(n)+a_{113} u_{1}^{2}(n) x_{3} \\
& +a_{123} u_{1}(n) u_{2}(n) x_{3} \\
& +o\left(\left(\left|u_{1}(n)\right|+\left|u_{2}(n)\right|+\left|x_{3}\right|\right)^{4}\right) \\
u_{2}( & +1) \\
= & b_{1} u_{1}(n)+b_{2} u_{2}(n)+b_{11} u_{1}^{2}(n) \\
& +b_{12} u_{1}(n) u_{2}(n)+b_{22} u_{2}^{2}(n) \\
& +b_{13} u_{1}(n) x_{3}+b_{23} u_{2}(n) x_{3}+b_{111} u_{1}^{3}(n) \\
& +b_{112} u_{1}^{2}(n) u_{2}(n)+b_{113} u_{1}^{2}(n) x_{3} \\
& +b_{123} u_{1}(n) u_{2}(n) x_{3}+b_{223} u_{2}^{2}(n) x_{3} \\
& +o\left(\left(\left|u_{1}(n)\right|+\left|u_{2}(n)\right|+\left|x_{3}\right|\right)^{4}\right)
\end{aligned}\right.
$$


where

$$
\begin{aligned}
& a_{i}=f_{x_{i}}\left(x_{1}^{*}, x_{2}^{*}, 0\right), \\
& b_{i}=g_{x_{i}}\left(x_{1}^{*}, x_{2}^{*}, 0\right), \\
& a_{i j}=f_{x_{i} x_{j}}\left(x_{1}^{*}, x_{2}^{*}, 0\right), \\
& b_{i j}=g_{x_{i} x_{j}}\left(x_{1}^{*}, x_{2}^{*}, 0\right), \\
& a_{i j k}=f_{x_{i} x_{j} x_{k}}\left(x_{1}^{*}, x_{2}^{*}, 0\right), \\
& b_{i j k}=g_{x_{i} x_{j} x_{k}}\left(x_{1}^{*}, x_{2}^{*}, 0\right), \quad i, j, k=1,2,3 .
\end{aligned}
$$

We define

$$
T=\left(\begin{array}{cc}
a_{2} & a_{2} \\
-1-a_{1} & \lambda_{2}-a_{1}
\end{array}\right)
$$

It is obvious that $T$ is invertible. Using the transformation

$$
\left(\begin{array}{l}
u_{1}(n) \\
u_{2}(n)
\end{array}\right)=T\left(\begin{array}{l}
\bar{x}_{1}(n) \\
\bar{x}_{2}(n)
\end{array}\right)
$$

the model (25) becomes

$$
\left\{\begin{array}{l}
\bar{x}_{1}(n+1)=-\bar{x}_{1}(n)+f_{1}\left(u_{1}(n), u_{2}(n), \delta^{*}\right), \\
\bar{x}_{2}(n+1)=\lambda_{2} \bar{x}_{2}(n)+g_{1}\left(u_{1}(n), u_{2}(n), \delta^{*}\right),
\end{array}\right.
$$

where the functions $f_{1}$ and $g_{1}$ denote the terms in the model $(28)$ in variables $\left(u_{1}(n), u_{2}(n), \delta^{*}\right)$ with the order at least two.

From the center manifold theorem given by Robinson (1999) and Guckenheimer (1983), we know that there exists a center manifold $W^{c}(0,0,0)$ of the model (28) at $(0,0)$ in a small neighborhood of $\delta^{*}=0$, which can be approximately described as follows:

$$
\begin{aligned}
& W^{c}(0,0,0) \\
& =\left\{\begin{array}{l}
\left(\bar{x}_{1}(n), \bar{x}_{2}(n), \delta^{*}\right) \in \mathbb{R}^{3}: \\
\bar{x}_{2}(n+1)=\bar{a}_{1} \bar{x}_{1}^{2}(n)+\bar{a}_{2} \bar{x}_{1}(n) \delta^{*} \\
\quad+o\left(\left(\left|\bar{x}_{1}(n)\right|+\left|\delta^{*}\right|\right)^{3}\right\},
\end{array}\right.
\end{aligned}
$$

where

$$
\begin{gathered}
\bar{a}_{1}=\frac{a_{2}\left[\left(1+a_{1}\right) a_{11}+a_{2} b_{11}\right]}{1-\lambda_{2}^{2}}+\frac{b_{22}\left(1+a_{1}\right)^{2}}{1-\lambda_{2}^{2}} \\
-\frac{\left(1+a_{1}\right)\left[a_{12}\left(1+a_{1}\right)+a_{2} b_{12}\right]}{1-\lambda_{2}^{2}} \\
\bar{a}_{2}=\frac{\left(1+a_{1}\right)\left[\left(1+a_{1}\right) a_{23}+a_{2} b_{23}\right]}{a_{2}\left(1+\lambda_{2}\right)^{2}} \\
-\frac{\left(1+a_{1}\right) a_{13}+a_{2} b_{13}}{\left(1+\lambda_{2}\right)^{2}} .
\end{gathered}
$$

We obtain that the model (28), which is restricted to center manifold $W^{c}(0,0,0)$, has the following form:

$$
\begin{aligned}
& \bar{x}_{1}(n+1) \\
&=-\bar{x}_{1}(n)+h_{1} \bar{x}_{1}^{2}(n)+h_{2} \bar{x}_{1}(n) \delta^{*} \\
& \quad+h_{3} \bar{x}_{1}^{2}(n) \delta^{*}+h_{4} \bar{x}_{1}(n) \delta^{* 2}+h_{5} \bar{x}_{1}^{3}(n) \\
& \quad+o\left(\left(\left|\bar{x}_{1}(n)\right|+\left|\delta^{*}\right|\right)^{3}\right) \\
& \triangleq F\left(\bar{x}_{1}(n), \delta^{*}\right),
\end{aligned}
$$

where

$$
\begin{aligned}
h_{1}= & \frac{\bar{a}_{2}\left[\left(\lambda_{2}-\bar{a}_{1}\right) a_{11}-\bar{a}_{2} b_{11}\right]}{1+\lambda_{2}}-\frac{b_{22}\left(1+\bar{a}_{1}\right)^{2}}{1+\lambda_{2}} \\
& -\frac{\left(1+\bar{a}_{1}\right)\left[\left(\lambda_{2}-\bar{a}_{1}\right) a_{12}-\bar{a}_{2} b_{12}\right]}{1+\lambda_{2}}
\end{aligned}
$$

$$
\begin{aligned}
h_{2}= & \frac{\left(\lambda_{2}-\bar{a}_{1}\right) a_{13}-b_{13} \bar{a}_{2}}{1+\lambda_{2}} \\
& -\frac{\left(1+\bar{a}_{1}\right)\left[\left(\lambda_{2}-\bar{a}_{1}\right) a_{23}-\bar{a}_{2} b_{23}\right]}{\bar{a}_{2}\left(1+\lambda_{2}\right)},
\end{aligned}
$$

$$
\begin{aligned}
h_{3}= & \frac{\left(\lambda_{2}-a_{1}\right) \bar{a}_{1} a_{13}-b_{13} a_{2}}{1+\lambda_{2}} \\
& +\frac{\left[\left(\lambda_{2}-a_{1}\right) a_{23}-a_{2} b_{23}\right] \bar{a}_{1}\left(\lambda_{2}-a_{1}\right)}{a_{2}\left(1+\lambda_{2}\right)} \\
& -\frac{\left(1+a_{1}\right)\left[\left(\lambda_{2}-a_{1}\right) a_{123}-a_{2} b_{123}\right]}{1+\lambda_{2}} \\
& +\frac{a_{2}\left[\left(\lambda_{2}-a_{1}\right) a_{113}-a_{2} b_{113}\right]}{1+\lambda_{2}}-\frac{b_{223}\left(1+a_{1}\right)^{2}}{1+\lambda_{2}} \\
& +\frac{2 a_{2} \bar{a}_{2}\left[\left(\lambda_{2}-a_{1}\right) a_{11}-a_{2} b_{11}\right]}{1+\lambda_{2}} \\
& -\frac{2 b_{22} \bar{a}_{2}\left(1+a_{1}\right)\left(\lambda_{2}-a_{1}\right)}{1+\lambda_{2}} \\
& +\frac{\bar{a}_{2}\left[\left(\lambda_{2}-a_{1}\right) a_{12}-a_{2} b_{12}\right]\left(\lambda_{2}-1-2 a_{1}\right)}{1+\lambda_{2}},
\end{aligned}
$$

$$
\begin{aligned}
h_{4}= & \frac{\bar{a}_{2}\left[\left(\lambda_{2}-a_{1}\right) a_{13}-b_{13} a_{2}\right]}{1+\lambda_{2}} \\
& +\frac{\left[\left(\lambda_{2}-a_{1}\right) a_{23}-a_{2} b_{23}\right] \bar{a}_{2}\left(\lambda_{2}-a_{1}\right)}{a_{2}\left(1+\lambda_{2}\right)} \\
& +\frac{2 a_{2} \bar{a}_{3}\left[\left(\lambda_{2}-a_{1}\right) a_{11}-a_{2} b_{11}\right]}{1+\lambda_{2}} \\
& +\frac{2 b_{22} \bar{a}_{3}\left[\left(1+a_{1}\right)\left(\lambda_{2}-a_{1}\right)\right]}{1+\lambda_{2}} \\
& +\frac{\left[\left(\lambda_{2}-a_{1}\right) a_{12}-a_{2} b_{12}\right]\left(\lambda_{2}-1-2 a_{1}\right) \bar{a}_{3}}{1+\lambda_{2}},
\end{aligned}
$$




$$
\begin{aligned}
h_{5}= & \frac{2 a_{2} \bar{a}_{1}\left[\left(\lambda_{2}-a_{1}\right) a_{11}-a_{2} b_{11}\right]}{1+\lambda_{2}} \\
& +\frac{2 b_{22} \bar{a}_{1}\left(1+a_{1}\right)\left(\lambda_{2}-a_{1}\right)}{1+\lambda_{2}} \\
& +\frac{\left[\left(\lambda_{2}-a_{1}\right) a_{11}-a_{2} b_{11}\right]\left(\lambda_{2}-1-2 a_{1}\right) \bar{a}_{1}}{1+\lambda_{2}} \\
& +\frac{\bar{a}_{2}^{2}\left[\left(\lambda_{2}-a_{1}\right) a_{111}-a_{2} b_{111}\right]}{1+\lambda_{2}} \\
& -\frac{\bar{a}_{2}\left(1+a_{1}\right)\left[\left(\lambda_{2}-a_{1}\right) a_{112}-a_{2} b_{112}\right]}{1+\lambda_{2}} .
\end{aligned}
$$

For flip bifurcation, we require that two discriminatory quantities $\alpha_{1}$ and $\alpha_{2}$ be nonzero,

$$
\begin{aligned}
\alpha_{1} & =\left.\left(\frac{\partial^{2} F}{\partial \bar{x}_{1} \partial \delta^{*}}+\frac{\partial F \partial^{2} F}{2 \partial \delta^{*} \partial \bar{x}_{1}^{2}}\right)\right|_{(0,0)}=h_{2}, \\
\alpha_{2} & =\left.\left(\frac{\partial^{3} F}{6 \partial^{3} \bar{x}_{1}}+\left(\frac{\partial^{2} F}{2 \partial \bar{x}_{1}^{2}}\right)^{2}\right)\right|_{(0,0)}=h_{5}+h_{1}^{2} .
\end{aligned}
$$

Finally, from the above analysis and Theorem 3.1 of Guckenheimer (1983, Chapter 7), we have the following result.

Theorem 1. If $\alpha_{1} \neq 0$ and $\alpha_{2} \neq 0$, then the model (2) undergoes flip bifurcation at $E_{2}\left(x_{1}^{*}, x_{2}^{*}\right)$ when the parameter $\delta$ varies in a small neighborhood of $\bar{\delta}$. Moreover, if $\alpha_{2}>0\left(\right.$ resp. $\left.\alpha_{2}<0\right)$, then the period-2 points that bifurcation from $E_{2}\left(x_{1}^{*}, x_{2}^{*}\right)$ are stable (resp. unstable).

Remark 1. For the equilibrium $E_{0}(0,0)$ of the model (2), by calculation we can obtain $\alpha_{1}=0$. Therefore, the conditions of Theorem 1 are not satisfied. Since $E_{0}(0,0)$ is always unstable for any $\delta>0$, the model (2) cannot undergo flip bifurcation at $E_{0}(0,0)$ when parameter $\delta$ varies in a neighborhood of $\delta=2 / \delta_{0}$.

In Section 7, we will give an example to show that flip bifurcation at $E_{2}\left(x_{1}^{*}, x_{2}^{*}\right)$ occurs under the conditions of Theorem 1.

\section{Hopf bifurcation}

In this section, we discuss the Hopf bifurcation of the model (2) at $E_{2}\left(x_{1}^{*}, x_{2}^{*}\right)$ when the parameters $\left(\delta_{1}, k, \beta, \delta_{0}, \gamma, \delta\right)$ vary in a small neighborhood of $F_{33}$. Taking parameters $\left(\delta_{1}, k, \beta, \delta_{0}, \gamma, \delta\right)=$ $\left(\delta_{11}, k_{1}, \beta_{1}, \delta_{01}, \gamma_{1}, \bar{\delta}\right) \in F_{33}$, and further giving $\delta$ a perturbation $\delta^{* *}$ at $\bar{\delta}$, the model (2) is described by

$$
\left\{\begin{aligned}
& x_{1}(n+1) \\
&= x_{1}(n)+\left(\bar{\delta}+\delta^{* *}\right) x_{1}(n)\left(1-x_{1}(n)\right. \\
&\left.-k_{1} x_{1}^{2}(n)-\frac{x_{2}(n)}{x_{1}^{2}(n)+\beta_{1}^{2}}\right) \\
& \triangleq \bar{f}\left(x_{1}(n), x_{2}(n), \delta^{* *}\right), \\
& x_{2}(n+1) \\
&=x_{2}(n)+\left(\bar{\delta}+\delta^{* *}\right) x_{2}(n)\left(-\delta_{01}\right. \\
&\left.\quad-\delta_{11} x_{2}(n)+\frac{\gamma_{1} x_{1}(n)}{x_{1}^{2}(n)+\beta_{1}^{2}}\right) \\
& \triangleq \bar{g}\left(x_{1}(n), x_{2}(n), \delta^{* *}\right) .
\end{aligned}\right.
$$

Let $u_{1}(n)=x_{1}(n)-x_{1}^{*}, u_{2}(n)=x_{2}(n)-x_{2}^{*}$ in the model (39). Then the equilibrium $E_{2}\left(x_{1}^{*}, x_{2}^{*}\right)$ is transformed into the origin. Further expanding $\bar{f}$ and $\bar{g}$ as a Taylor series at $\left(u_{1}, u_{2}\right)=(0,0)$ to the third order, we have

$$
\left\{\begin{aligned}
u_{1}( & +1) \\
= & c_{1} u_{1}(n)+c_{2} u_{2}(n)+c_{11} u_{1}^{2}(n) \\
& +c_{12} u_{1}(n) u_{2}(n)+c_{22} u_{2}^{2}(n) \\
& +c_{111} u_{1}^{3}(n)+c_{112} u_{1}^{2}(n) u_{2}(n) \\
& +c_{122} u_{1}(n) u_{2}^{2}(n)+c_{22} u_{2}^{3}(n) \\
& +o\left(\left(\left|u_{1}(n)\right|+\left|u_{2}(n)\right|\right)^{4}\right), \\
u_{2}( & +1) \\
= & d_{1} u_{1}(n)+d_{2} u_{2}(n)+d_{11} u_{1}^{2}(n) \\
& +d_{12} u_{1}(n) u_{2}(n)+d_{22} u_{2}^{2}(n) \\
& +d_{111} u_{1}^{3}(n)+d_{112} u_{1}^{2}(n) u_{2}(n) \\
& +d_{122} u_{1}(n) u_{2}^{2}(n)+d_{222} u_{2}^{3}(n) \\
& +o\left(\left(\left|u_{1}(n)\right|+\left|u_{2}(n)\right|\right)^{4}\right),
\end{aligned}\right.
$$

where

$$
\begin{aligned}
c_{i} & =\bar{f}_{x_{i}}\left(x_{1}^{*}, x_{2}^{*}, 0\right), \\
d_{i} & =\bar{g}_{x_{i}}\left(x_{1}^{*}, x_{2}^{*}, 0\right), \\
c_{i j} & =\bar{f}_{x_{i} x_{j}}\left(x_{1}^{*}, x_{2}^{*}, 0\right), \\
d_{i j} & =\bar{g}_{x_{i} x_{j}}\left(x_{1}^{*}, x_{2}^{*}, 0\right), \\
c_{i j k} & =\bar{f}_{x_{i} x_{j} x_{k}}\left(x_{1}^{*}, x_{2}^{*}, 0\right), \\
d_{i j k} & =\bar{g}_{x_{i} x_{j} x_{k}}\left(x_{1}^{*}, x_{2}^{*}, 0\right), \quad i, j, k=1,2 .
\end{aligned}
$$

Note that the characteristic equation associated with the linearization of the model $(40)$ at $\left(u_{1}(n), u_{2}(n)\right)=$ $(0,0)$ is given by

$$
\lambda^{2}+p\left(\delta^{* *}\right) \lambda+q\left(\delta^{* *}\right)=0,
$$


where

$$
\begin{aligned}
& p\left(\delta^{* *}\right)=-2-G\left(\delta^{* *}+\bar{\delta}\right), \\
& q\left(\delta^{* *}\right)=1+G\left(\delta^{* *}+\bar{\delta}\right)+H\left(\delta^{* *}+\bar{\delta}\right)^{2} .
\end{aligned}
$$

The roots of the characteristic equation (41) are

$$
\lambda_{1,2}\left(\delta^{* *}\right)=\frac{1}{2}\left(-p\left(\delta^{* *}\right) \pm i \sqrt{4 q\left(\delta^{* *}\right)-p^{2}\left(\delta^{* *}\right)} .\right.
$$

We have

$$
\left|\lambda_{1,2}\left(\delta^{* *}\right)\right|=\left(q\left(\delta^{* *}\right)\right)^{\frac{1}{2}}
$$

From $\left|\lambda_{1,2}\left(\delta^{* *}\right)\right|=1$, when $\delta^{* *}=0$ we have

$$
q(0)=1+G \bar{\delta}+H \bar{\delta}^{2}=1 .
$$

Hence, $H \bar{\delta}=-G$. Consequently,

$$
l=\left.\frac{\mathrm{d}\left|\lambda_{1,2}\left(\delta^{* *}\right)\right|}{\mathrm{d} \delta^{* *}}\right|_{\delta^{* *}=0}=-\frac{G}{2}>0 .
$$

In addition, it is required that, when $\delta^{* *}=0, \lambda_{1,2}^{m} \neq 1$ for $m=1,2,3,4$, which is equivalent to $p(0) \neq-2,0,1,2$.

Note that $\Delta=p^{2}(0)-4 q(0)<0$ and then $p(0) \neq$ $-2,2$. We only prove that $p(0) \neq 0,1$, and we have $p(0)=-2-G \bar{\delta} \neq 0,1$. Then $-G \bar{\delta} \neq 2,3$, and hence

$$
\bar{\delta} x_{1}^{*}+2 k_{1} \bar{\delta} x_{1}^{* 2}+\bar{\delta} \delta_{11} x_{2}^{*}-\frac{2 \bar{\delta} x_{1}^{* 2} x_{2}^{*}}{\left(x_{1}^{* 2}+\beta_{1}^{2}\right)^{2}} \neq 2,3 .
$$

Therefore, eigenvalues $\lambda_{1,2}$ do not lie in the intersection of the unit circle with the coordinate axes when $\delta^{* *}=0$ and (47) holds.

Let

$$
\begin{aligned}
& \alpha=1-\frac{1}{2} \bar{\delta}\left[x^{*}+2 k x^{* 2}+\delta_{1} y^{*}-\frac{2 x^{* 2} y^{*}}{\left(x^{* 2}+\beta^{2}\right)^{2}}\right] \\
& \omega=\delta_{12} \sqrt{4 H-G}
\end{aligned}
$$

and

$$
T=\left(\begin{array}{ll}
0 & 1 \\
\omega & \alpha
\end{array}\right)
$$

Use the transformation

$$
\left(\begin{array}{l}
u_{1}(n) \\
u_{2}(n)
\end{array}\right)=T\left(\begin{array}{l}
\widehat{x}_{1}(n) \\
\widehat{x}_{2}(n)
\end{array}\right) .
$$

Then the model (40) becomes

$$
\left\{\begin{array}{l}
\widehat{x}_{1}(n+1)=\alpha \widehat{x}_{1}(n)-\omega \widehat{x}_{2}(n)+f_{2}\left(\widehat{x}_{1}(n), \widehat{x}_{2}(n)\right), \\
\widehat{x}_{2}(n+1)=\omega \widehat{x}_{1}(n)+\alpha \widehat{x}_{2}(n)+g_{2}\left(\widehat{x}_{1}(n), \widehat{x}_{2}(n)\right),
\end{array}\right.
$$

where the functions $f_{2}$ and $g_{2}$ denote the terms in the model (52) with the order at least two in variables $\left(\widehat{x}_{1}(n), \widehat{x}_{2}(n)\right)$.

In order to undergo Hopf bifurcation, we require that the following discriminatory quantity $a$ be nonzero:

$$
\begin{aligned}
a= & -\operatorname{Re}\left[\frac{(1-2 \bar{\lambda}) \bar{\lambda}^{2}}{1-\lambda} \xi_{11} \xi_{20}\right]-\frac{1}{2}\left\|\xi_{11}\right\|^{2} \\
& -\left\|\xi_{02}\right\|^{2}+\operatorname{Re}\left(\bar{\lambda} \xi_{21}\right)
\end{aligned}
$$

where

$$
\begin{aligned}
\xi_{20}= & \frac{1}{8} \alpha\left(2 d_{22}-\alpha c_{22}-c_{12}+4 \omega c_{22}\right) \\
& +\frac{1}{4} \omega c_{12}+\frac{1}{8} \alpha i\left(4 \omega c_{22}-2 c_{12}-2 \alpha c_{22}\right) \\
& +\frac{1}{8} i\left(4 \omega d_{22}+2 \omega^{2} c_{22}-2 c_{11}\right) \\
& +\frac{\alpha c_{11}-2 d_{11}}{4 \omega}+\frac{\alpha^{3} c_{22}-\alpha^{2} d_{22}}{4 \omega} \\
& +\frac{1}{8} d_{12}-\frac{\alpha^{2} c_{12}-\alpha d_{12}}{4 \omega}, \\
\xi_{11}= & \frac{1}{2} \omega\left(d_{22}-\alpha c_{22}\right)+\frac{1}{2} i\left(\omega^{2} c_{22}+c_{11}\right. \\
& \left.+\alpha c_{12}+\alpha^{2} c_{22}\right)+\frac{d_{11}-\alpha c_{11}}{2 \omega} \\
& +\frac{\alpha d_{12}-\alpha^{2} c_{12}}{2 \omega}-\frac{\alpha^{2} d_{22}-\alpha^{3} c_{22}}{\omega}, \\
\xi_{02}= & \frac{1}{4} \omega\left(2 \alpha c_{22}+c_{12}+d_{22}\right) \\
& +\frac{1}{4} i\left(d_{12}+2 \alpha d_{22}-2 \alpha c_{12}-c_{11}\right) \\
& -\frac{d_{11}-\alpha c_{11}}{4 \omega}-\frac{\alpha d_{12}-\alpha^{2} c_{12}}{4 \omega} \\
& +\frac{1}{4} c_{22} i\left(\omega^{2}-3 \alpha^{2}\right)+\frac{\alpha^{2} d_{22}-\alpha^{3} c_{22}}{4 \omega}
\end{aligned}
$$

$$
\begin{aligned}
\xi_{21}= & \frac{3}{8} d_{222}\left(\omega^{2}+\alpha^{2}\right)+\frac{1}{8} d_{112}+\frac{1}{4} \alpha c_{112} \\
& +\frac{1}{4} \alpha d_{122}+c_{122}\left(\frac{1}{8} \omega^{2}+\frac{3}{8} \alpha^{2}-\frac{1}{4} \alpha\right) \\
& +\frac{3}{8} c_{111}+\frac{3}{8} c_{222} i\left(\omega^{2}+2 \alpha^{2}\right)+\frac{3}{8} \alpha \omega c_{122} i \\
& -\frac{1}{8} \omega d_{122} i-\frac{3}{8} \alpha \omega d_{222} i-\frac{3 d_{111}-3 \alpha c_{111}}{8 \omega} i \\
& -\frac{3 \alpha d_{112}-3 \alpha^{2} c_{112}}{8 \omega} i-\frac{3 \alpha^{2} d_{122}-3 \alpha^{3} c_{122}}{8 \omega} i \\
& -\frac{3 \alpha^{3} d_{222}-3 \alpha^{4} c_{222}}{8 \omega} i
\end{aligned}
$$

From the above analysis and Theorem 3.5.2 of Guckenheimer (1983), we have the following result. 
Theorem 2. If the condition (47) holds and $a \neq 0$, then the model (2) undergoes Hopf bifurcation at $E_{2}\left(x_{1}^{*}, x_{2}^{*}\right)$ when the parameter $\delta^{* *}$ varies in a small neighborhood of the origin. Moreover, if $a<0$ (resp. $a>0$ ), then an attracting (resp. repelling) invariant closed curve bifurcates from $E_{2}\left(x_{1}^{*}, x_{2}^{*}\right)$ for $\delta^{* *}>0$ (resp., $\left.\delta^{* *}<0\right)$.

In Section 7, we will give an example to show Hopf bifurcation occurs at $E_{2}\left(x_{1}^{*}, x_{2}^{*}\right)$ under the conditions of Theorem 2.

\section{Saddle-node bifurcation}

Finally, we discuss saddle-node bifurcation of model (2) at $E_{2}\left(x_{1}^{*}, x_{2}^{*}\right)$ when parameters $\left(\delta_{1}, k, \beta, \delta_{0}, \gamma, \delta\right)$ vary in a small neighborhood of $F_{34}$. Taking parameters $\left(\delta_{1}, k, \beta, \delta_{0}, \gamma, \delta\right)=\left(\delta_{11}, k_{1}, \beta_{1}, \delta_{01}, \gamma_{1}, \bar{\delta}\right) \in F_{34}$, and further giving $\delta_{1}$ a perturbation $\delta_{1}^{*}$ at $\delta_{11}$, the model (2) is described by

$$
\left\{\begin{aligned}
x_{1}(n+1) & \\
= & x_{1}(n)+\bar{\delta} x_{1}(n)\left(1-x_{1}(n)\right. \\
& \left.-k_{1} x_{1}^{2}(n)-\frac{x_{2}(n)}{x_{1}^{2}(n)+\beta_{1}^{2}}\right) \\
\triangleq & \widehat{f}\left(x_{1}(n), x_{2}(n), x_{3}\right), \\
x_{2} & (n+1) \\
= & x_{2}(n)+\bar{\delta} x_{2}(n)\left[-\delta_{01}\right. \\
& \left.-\left(\delta_{11}+\delta_{1}^{*}\right) x_{2}(n)+\frac{\gamma_{1} x_{1}(n)}{x_{1}^{2}(n)+\beta_{1}^{2}}\right] \\
\triangleq & \widehat{f}\left(x_{1}(n), x_{2}(n), x_{3}\right),
\end{aligned}\right.
$$

where $\left|\delta_{1}^{*}\right| \ll 1$, and $x_{3}=\delta_{1}^{*}$.

Let $u_{1}(n)=x_{1}(n)-x_{1}^{*}, u_{2}(n)=x_{2}(n)-x_{2}^{*}$. Then the equilibrium $E_{2}\left(x_{1}^{*}, x_{2}^{*}\right)$ is transformed into the origin, and further expanding $\widehat{f}$ and $\widehat{g}$ as the Taylor series at $\left(u_{1}, u_{2}, x_{3}\right)=(0,0,0)$ to the second order, the model (58) becomes

$$
\left\{\begin{aligned}
u_{1}(n+1) & \\
= & e_{1} u_{1}(n)+e_{2} u_{2}(n) \\
& +e_{11} u_{2}^{2}(n)+e_{12} u_{1}(n) u_{2}(n) \\
& +o\left(\left(\left|u_{1}(n)\right|+\left|u_{2}(n)\right|+\left|x_{3}\right|\right)^{3}\right), \\
u_{2}(n+1) & \\
= & k_{1} u_{1}(n)+k_{2} u_{2}(n) \\
& +k_{3} x_{3}+k_{11} u_{1}^{2}(n)+k_{12} u_{1}(n) u_{2}(n) \\
& +k_{22} u_{2}^{2}(n)+k_{23} u_{2}(n) x_{3} \\
& +o\left(\left(\left|u_{1}(n)\right|+\left|u_{2}(n)\right|+\left|x_{3}\right|\right)^{3}\right),
\end{aligned}\right.
$$

where

$$
\begin{aligned}
e_{i} & =\widehat{f}_{x_{i}}\left(x_{1}^{*}, x_{2}^{*}, 0\right), \\
k_{i} & =\widehat{g}_{x_{i}}\left(x_{1}^{*}, x_{2}^{*}, 0\right), \\
e_{i j} & =\widehat{f}_{x_{i} x_{j}}\left(x_{1}^{*}, x_{2}^{*}, 0\right), \\
k_{i j} & =\widehat{g}_{x_{i} x_{j}}\left(x_{1}^{*}, x_{2}^{*}, 0\right), \quad i, j=1,2,3 .
\end{aligned}
$$

Let

$$
T=\left(\begin{array}{cc}
\bar{a}_{11} & 1 \\
1 & \bar{a}_{22}
\end{array}\right)
$$

where

$$
\begin{aligned}
& \bar{a}_{11}=\frac{x_{3}\left(x_{1}^{* 2}+\beta_{1}^{2}\right)^{2}}{\left(\beta_{1}^{2}-x_{1}^{* 2}\right) \gamma_{1}}, \\
& \bar{a}_{22}=\frac{x_{3} x_{2}^{*}\left(x_{1}^{* 2}+\beta_{1}^{2}\right)}{x_{1}^{*}} .
\end{aligned}
$$

Use the transformation

$$
\left(\begin{array}{l}
u(n) \\
v(n)
\end{array}\right)=T\left(\begin{array}{l}
\widetilde{x}_{1}(n) \\
\widetilde{x}_{2}(n)
\end{array}\right) .
$$

Then the model (59) becomes

$$
\left\{\begin{array}{l}
\widetilde{x}_{1}(n+1) \\
=\widetilde{x}_{1}(n)-\frac{k_{3}}{|T|} \delta_{1}^{*}+f_{3}\left(\widetilde{x}_{1}(n), \widetilde{x}_{2}(n), \delta_{1}^{*}\right), \\
\widetilde{x}_{2}(n+1) \\
=\lambda_{2} \widetilde{x}_{2}(n)+\frac{k_{3} \bar{a}_{11}}{|T|} \delta_{1}^{*} \\
\quad+g_{3}\left(\widetilde{x}_{1}(n), \widetilde{x}_{2}(n), \delta_{1}^{*}\right),
\end{array}\right.
$$

where the functions $f_{3}$ and $g_{3}$ denote the terms in the model (63) with the order at least two in variables $\left(\widetilde{x}_{1}(n), \widetilde{x}_{2}(n), \delta_{1}^{*}\right)$.

Applying the center manifold theorem given by Guckenheimer (1983) and Kuznetsov (1998), we obtain that there exists a center manifold $W^{c}(0,0,0)$ of the model $(63)$ at $(0,0)$ in a small neighborhood of $\delta_{1}^{*}=0$, which can be approximately described as follows:

$$
\begin{aligned}
W^{c}(0,0,0) & \\
= & \left\{\left(\widetilde{x}_{1}, \widetilde{x}_{2}, \delta_{1}^{*}\right) \in \mathbb{R}^{3}: \widetilde{x}_{2}(n+1)=\widehat{a}_{0} \delta_{1}^{*}\right. \\
& +\widehat{a}_{1} \widetilde{x}_{1}(n) \delta_{1}^{*}+\widehat{a}_{2} \widetilde{x}_{1}(n)^{2}+\widehat{a}_{3} \delta_{1}^{* 2} \\
& \left.+o\left(\left(\left|\widetilde{x}_{1}(n)\right|+\left|\delta_{1}^{*}\right|\right)^{3}\right)\right\},
\end{aligned}
$$

where

$$
\widehat{a}_{0}=\frac{1}{1-\lambda_{2}} \frac{k_{3} \bar{a}_{11}}{|T|}
$$




$$
\begin{aligned}
\widehat{a}_{1}= & \frac{1}{1-\lambda_{2}}\left[2 \widehat{a}_{0} \bar{a}_{11} \frac{\bar{a}_{11} k_{11}-e_{11}}{|T|}\right. \\
& +\widehat{a}_{0}\left(1+\bar{a}_{11} \bar{a}_{22}\right) \frac{\bar{a}_{11} k_{12}-e_{12}}{|T|} \\
& \left.+2 \widehat{a}_{0} \bar{a}_{22} \frac{\bar{a}_{11} k_{22}}{|T|}+\frac{\bar{a}_{11} k_{23}}{|T|}\right], \\
\widehat{a}_{2}= & \frac{\bar{a}_{11}}{1-\lambda_{2}}\left[\bar{a}_{11} \frac{k_{11}-e_{11}}{|T|}+\frac{k_{22}}{|T|}\right. \\
& \left.+\bar{a}_{11}^{2} \frac{\bar{a}_{11} k_{12}-e_{12}}{|T|}\right], \\
\widehat{a}_{3}= & \frac{\widehat{a}_{0}}{1-\lambda_{2}}\left[\frac{\bar{a}_{11} k_{11}-e_{11}}{|T|}+\frac{k_{22} \bar{a}_{11} \bar{a}_{22}^{2}}{|T|}\right. \\
& \left.+\bar{a}_{22} \frac{\bar{a}_{11} k_{12}-e_{12}}{|T|}+\frac{\bar{a}_{22} \bar{a}_{11} k_{23}}{|T|}\right] .
\end{aligned}
$$

Further, we have that the model (63) restricted to center manifold $W^{c}(0,0,0)$ has the following form:

$$
\begin{aligned}
& \widetilde{x}_{1}(n+1) \\
& =\widetilde{x}_{1}(n)+l_{0} \delta_{1}^{*}+l_{1} \widetilde{x}_{1}(n)^{2}+l_{2} \widetilde{x}_{1}(n) \delta_{1}^{*} \\
& \quad+l_{3} \delta_{1}^{* 2}+o\left(\left(\left|\widetilde{x}_{1}(n)\right|+\left|\delta_{1}^{*}\right|\right)^{3}\right) \\
& \triangleq \\
& \triangleq
\end{aligned}
$$

where

$$
\begin{gathered}
l_{0}=-\frac{k_{3}}{|T|}, \\
l_{1}=\frac{\bar{a}_{22} e_{11}-k_{11}}{|T|} \bar{a}_{11}^{2}+\frac{\bar{a}_{22} e_{12}-k_{12}}{|T|} \bar{a}_{11}, \\
l_{2}=2 \widehat{a}_{0} \bar{a}_{11} \frac{\bar{a}_{22} e_{11}-k_{11}}{|T|}-\frac{2 \widehat{a}_{0} \bar{a}_{22} k_{22}}{|T|} \\
+\widehat{a}_{0}\left(1+\bar{a}_{1} 1 \bar{a}_{22}\right) \frac{\bar{a}_{22} e_{12}-k_{12}}{|T|}-\frac{k_{23}}{|T|}, \\
l_{3}=\frac{\bar{a}_{22} e_{11}-k_{11}}{|T| \widehat{a}_{0}}-\frac{\widehat{a}_{0} \bar{a}_{22}^{2} k_{22}}{|T|} \\
+\frac{\bar{a}_{22} e_{12}-k_{12}}{|T|} \widehat{a}_{0} \bar{a}_{22}-\frac{\widehat{a}_{0} \bar{a}_{22} k_{23}}{|T|} .
\end{gathered}
$$

In order to undergo saddle-node bifurcation, we require that the discriminatory quantities $\eta_{i}(i=1,2,3,4)$ be nonzero,

$$
\begin{aligned}
& \eta_{1}=\left.\frac{\partial \widehat{F}}{\partial \delta_{1}^{*}}\right|_{(0,0)}=l_{0} \neq 0, \\
& \eta_{2}=\left.3 \frac{\partial \widehat{F}}{\partial \widetilde{x}_{1}}\right|_{(0,0)}=1 \neq 0,
\end{aligned}
$$

$$
\begin{gathered}
\eta_{3}=\left.\frac{\partial^{2} \widehat{F}}{\partial \widetilde{x}_{1} \partial \delta_{1}^{*}}\right|_{(0,0)}=l_{2} \neq 0, \\
\eta_{4}=\left.\frac{\partial^{2} \widehat{F}}{\partial \widetilde{x}_{1}^{2}}\right|_{(0,0)}=l_{1} \neq 0 .
\end{gathered}
$$

From the above analysis and applying Theorem 4.1 given by Kuznetsov (1998, Chapter 4), we have the following theorem.

Theorem 3. If $\eta_{i}(i=1,3,4) \neq 0$, then the model (2) undergoes saddle-node bifurcation at $E_{2}\left(x_{1}^{*}, x_{2}^{*}\right)$ when the parameter $\delta_{1}^{*}$ varies in a small neighborhood of the origin. Moreover, if $\eta_{i}(i=1,3,4)>0$ (resp. $\eta_{i}(i=1,3,4)<$ $0)$ and $\delta_{1}^{*}>0\left(\right.$ resp. $\left.\delta_{1}^{*}<0\right)$, then new equilibria occur (resp. equilibria overlap).

In Section 7, we will give an example to show that saddle-node bifurcation occurs at $E_{2}\left(x_{1}^{*}, x_{2}^{*}\right)$ under the conditions of Theorem 3.

\section{Chaos control}

Unstable fluctuations, bifurcations and chaos have always been regarded as unfavorable phenomena in biology, so they are harmful for the breeding of biological population. Naturally, we need take action to stabilize the latter.

In this section, to eliminate the unstable periodic orbits or the chaotic orbits of the model (2), we will introduce the OGY chaos control method given by Ott et al. (1990), Grebogi et al (1983) as well as Scholl and Schuster (2008) to the model (2). We will design an OGY controller to stabilize the unstable periodic orbits embedded in the chaotic attractor of the model (2) to a positive equilibrium.

Consider the following corresponding controlled form of (2):

$$
\left\{\begin{array}{l}
x_{1}(n+1) \\
=x_{1}(n)+\left(( \delta ^ { * } + p ) x _ { 1 } ( n ) \left(1-x_{1}(n)\right.\right. \\
\left.\left.\quad-k x_{1}(n)^{2}-\frac{x_{2}(n)}{x_{1}(n)^{2}+\beta^{2}}\right)\right) \\
x_{2}(n+1) \\
=x_{2}(n)+\left(\delta^{*}+p\right) x_{2}(n)\left(-\delta_{0}\right. \\
\left.\quad-\delta_{1} x_{2}(n)+\frac{\gamma x_{1}(n)}{x_{1}(n)^{2}+\beta^{2}}\right)
\end{array}\right.
$$

where $p$ is chosen as the control adjusted signal. Here, we always assume that the model (2) has unstable periodic orbits embedded in the chaotic attractor when $\delta=\delta^{*}$.

For simplicity, we only discuss the control law of period-1 orbits; the other cases (for example, higher period) are similar to this case. We will stabilize the unstable periodic orbits embedded in the chaotic attractor 
of the model (2) to equilibrium $E_{2}\left(x_{1}^{*}, x_{2}^{*}\right)$. Choose the response vector $w$ as follows:

$$
w=\operatorname{det}\left(I-J\left(E_{2}\right)\right)\left(\begin{array}{l}
1 \\
1
\end{array}\right),
$$

where $I$ denotes the identity matrix. Let $\lambda_{1}$ and $\lambda_{2}$ be eigenvalues of $J\left(E_{2}\right)$, and $\lambda_{1}\left(\lambda_{2}\right)$ correspond to the unstable manifold (stable manifold). Then $\left|\lambda_{1}\right|>1$ $\left(\left|\lambda_{2}\right|<1\right)$.

The unstable orientation of $E_{2}\left(x_{1}^{*}, x_{2}^{*}\right)$ is denoted by $\rho_{1}$ and orthogonal dual vector $\rho_{2}$ :

$$
\begin{gathered}
\rho_{1}=\left(\begin{array}{c}
1 \\
\frac{\left(\beta^{2}-x_{1}^{* 2}\right) \gamma \delta x_{2}^{*}}{\left(x_{1}^{* 2}+\beta^{2}\right)^{2}\left(\lambda_{1}-1+\delta \delta_{1} x_{2}^{*}\right)}
\end{array}\right), \\
\rho_{2}=\left(\begin{array}{c}
1 \\
-\frac{\delta x_{1}^{*}}{\left(x_{1}^{* 2}+\beta^{2}\right)\left(\lambda_{1}-1+\delta \delta_{1} x_{2}^{*}\right)}
\end{array}\right),
\end{gathered}
$$

and the control adjusted signal can be written as

$$
p=\frac{\lambda_{1} \rho_{2}^{T}}{\left(\lambda_{1}-1\right) \rho_{2}^{T} w}\left(\begin{array}{c}
x_{1}(n)-x_{1}^{*} \\
x_{2}(n)-x_{2}^{*}
\end{array}\right) .
$$

Choose the allowable range of parameter variation $\epsilon$ (for example, $\epsilon=0.08)$ ). Then we obtain the control condition

$$
\left|\Delta S_{x_{1}}^{x_{2}}\right|<\left(1-\frac{1}{\lambda_{1}}\right) \rho_{2}^{T} w \epsilon
$$

where

$$
\Delta S_{x_{1}}^{x_{2}}=\rho_{2}^{T}\left(\begin{array}{c}
x_{1}(n)-x_{1}^{*} \\
x_{2}(n)-x_{2}^{*}
\end{array}\right) .
$$

If the model (78) satisfies the control condition, we start control. Applying the results given by Ott et al. (1990), Grebogi et al (1983) as well as Scholl and Schuster (2008), we have the following theorem.

Theorem 4. If the controlled model (78) satisfies the control condition

$$
\left|\Delta S_{x_{1}}^{x_{2}}\right|<\left(1-\frac{1}{\lambda_{1}}\right) \rho_{2}^{T} \omega \epsilon
$$

$0<\epsilon \ll 1$ defines the allowable range of parameter variation, and the controller adjusted signal is

$$
p=\left\{\begin{array}{c}
0 \quad \text { if }\left|\frac{\lambda_{1}}{\left(\lambda_{1}-1\right) \rho_{2} \omega} \Delta S_{x_{1}}^{x_{2}}\right|<\epsilon, \\
\frac{\lambda_{1} \rho_{2}^{T}}{\left(\lambda_{1}-1\right) \rho_{2}^{T} w}\left(\begin{array}{c}
x_{1}-x_{1}^{*} \\
x_{2}-x_{2}^{*}
\end{array}\right) \text { otherwise, }
\end{array}\right.
$$

then this controller can stabilize the unstable periodic orbits embedded in the chaotic attractor to a positive equilibrium.
For the model (2), when to start controlling the unstable periodic orbits embedded in the chaotic attractor is quite important. Ott et al. (1990) and Grebogi et al. $(1986 ; 1987)$ gave detailed expressions for general OGY chaos control. The time of starting control usually depends on the control adjusted signal $p$. When time $t$ is large enough, $p$ has the following exponential probability distribution:

$$
p(t) \propto \exp \left(-\frac{t}{\langle t\rangle}\right)
$$

where $\langle t\rangle$ has to do with $\delta$ and presents the average time of chaos. Particularly, when $\delta$ is quite small, we further have

$$
\langle t\rangle \propto \delta^{-\gamma}
$$

where

$$
\gamma=1+\frac{1}{2} \frac{\ln \lambda_{1}}{\ln \left|\lambda_{2}\right|^{-1}}
$$

Therefore, the time of starting control can be determined by $\delta$ and $\gamma$.

\section{Numerical simulations}

In this section, we give the bifurcation diagrams, phase portraits of the model (2) to confirm the above theoretical analysis and show new interesting complex dynamical behaviors by using numerical simulations. Furthermore, we have performed some numerical simulations to see how the OGY chaos control method stabilizes the unstable periodic orbits embedded in the chaotic attractor. The parameters are considered for the following four cases.

Case 1. Varying $\delta$ in the range $0.8 \leq \delta \leq 2.2$, and fixing $\delta_{1}=0.32, k=2.2, \beta=0.5, \delta_{0}=0.2, \gamma=0.51$. When we evaluate Eqns. (4), the model (2) has a unique positive equilibrium $E_{2}(0.1434,0.2195)$. When $\delta=$ 0.8565 , the Jacobian matrix $J\left(E_{2}\right)$ has two eigenvalues $\lambda_{ \pm}=0.9226 \pm i 0.3858$ with $\left|\lambda_{ \pm}\right|=1$. Hence, $\left(\delta_{1}, k, \beta, \delta_{0}, \gamma, \delta\right)=(0.32,2.2,0.5,0.2,0.51,0.8565) \in$ $F_{33}$. Further, by computing (53), we can obtain that $a=-0.1235$. This illustrates Theorem 2 .

From Fig. 1, we observe that the equilibrium $E_{2}$ of the model (2) is stable for $\delta<0.8565$ and loses its stability at $\delta=0.8565$, and that an invariant circle appears when $\delta$ exceeds 0.8565 .

Figure 1 clearly depicts how a smooth invariant circle bifurcates from the stable equilibrium $E_{2}$. When $\delta$ exceeds 0.8565 , there appears a circular curve enclosing $E_{2}$, and its radius becomes larger with the growth of $\delta$ when $\delta$ reaches certain values. For instance, $\delta=1.84$, the circle disappears and the period-3, 6, 9, 12, 20, 63, 70, 112 windows within the chaotic regions and boundary crisis emerges at $\delta=2.1813$. Further, from Fig. 1 we observe 


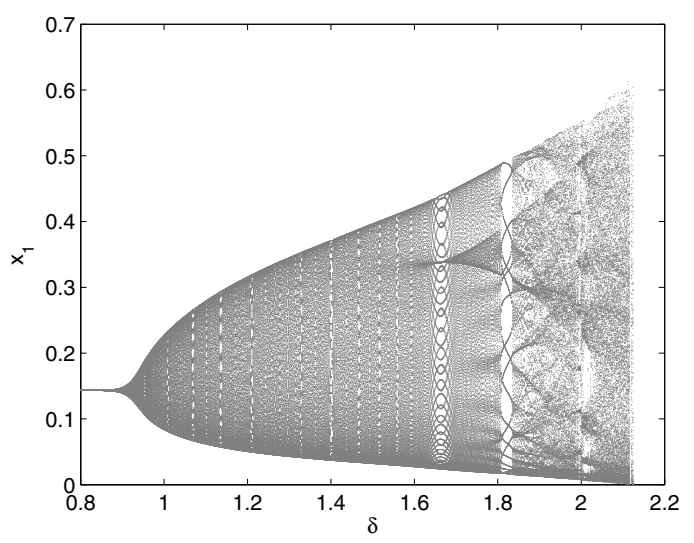

Fig. 1. Hopf bifurcation of the model (2) in the $\left(\delta, x_{1}\right)$ plane, and initial values $\left(x_{10}, x_{20}\right)=(0.3,0.1)$.

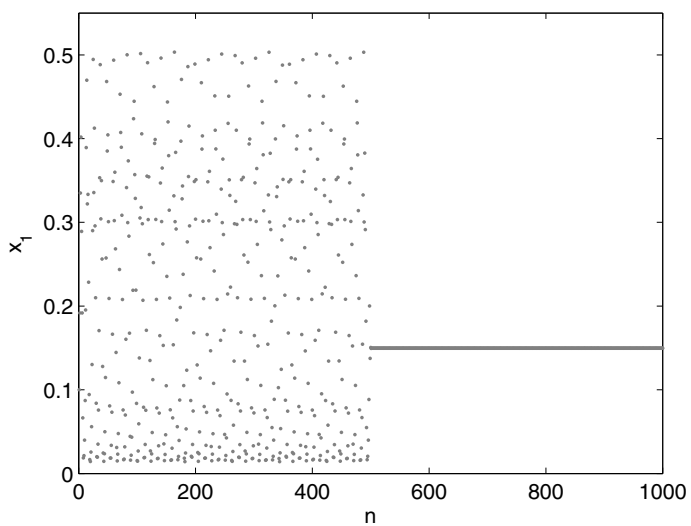

Fig. 2. Time responses for the state $x_{1}$ of the controlled model (78) in the $\left(n, x_{1}\right)$ plane with $\delta=1.86$, and initial values $\left(x_{10}, x_{20}\right)=(0.3,0.1)$

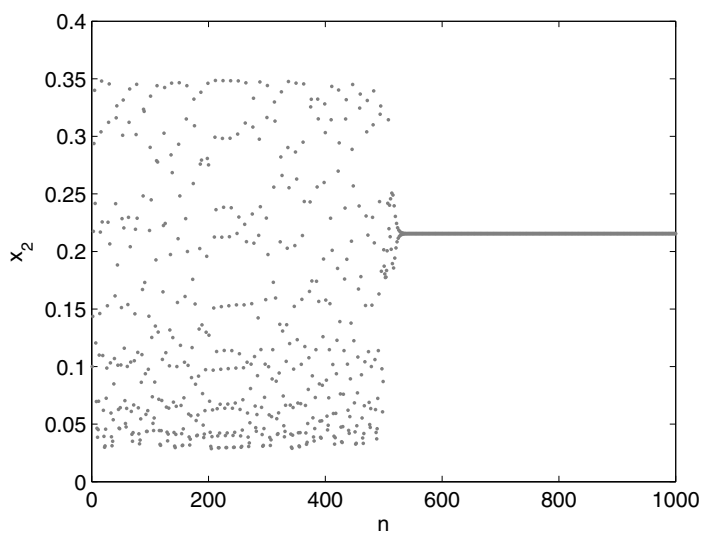

Fig. 3. Time responses for the state $x_{2}$ of the controlled model (78) in the $\left(n, x_{2}\right)$ plane with $\delta=1.86$, and initial values $\left(x_{10}, x_{20}\right)=(0.3,0.1)$. the number of predators decreases due to the increased ability of prey and the population of the predators is extinct at last.

We have performed some numerical simulations to see how the OGY controller stabilizes the unstable periodic orbits embedded in the chaotic attractor. Parameter values are fixed as $\left(\delta_{1}, k, \beta, \delta_{0}, \gamma, \delta\right)=$ $(0.32,2.2,0.5,0.2,0.51,0.933)$. Figures 2 and 3 show that, when $n<500$, we do not apply control to the model (2) (that is, $p \equiv 0$ ), and (2) presents chaotic behavior. When $n>500$, the chaotic orbits fall into a small neighborhood of the desired equilibrium, we start control, so that the chaotic orbit is quickly stabilized to $E_{2}$.

Case 2. Varying $\delta$ in the range $1.2 \leq \delta \leq 2$, and fixing $\delta_{1}=0.8, k=2.5, \beta=0.4, \delta_{0}=0.6, \gamma=0.5$. By evaluating Eqn. (4), the model (2) has a unique positive equilibrium $E_{2}(0.4391,0.0279)$. When $\delta=$

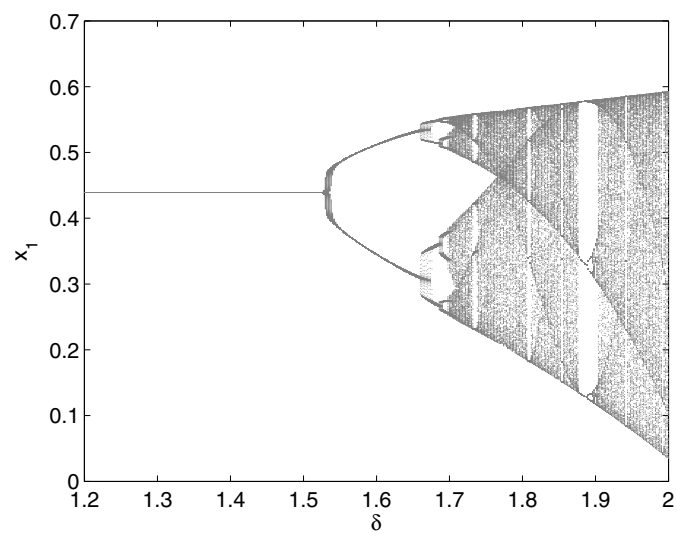

Fig. 4. Flip bifurcation of the model (2) in the $\left(\delta, x_{1}\right)$ plane, and initial values $\left(x_{10}, x_{20}\right)=(0.3,0.1)$.

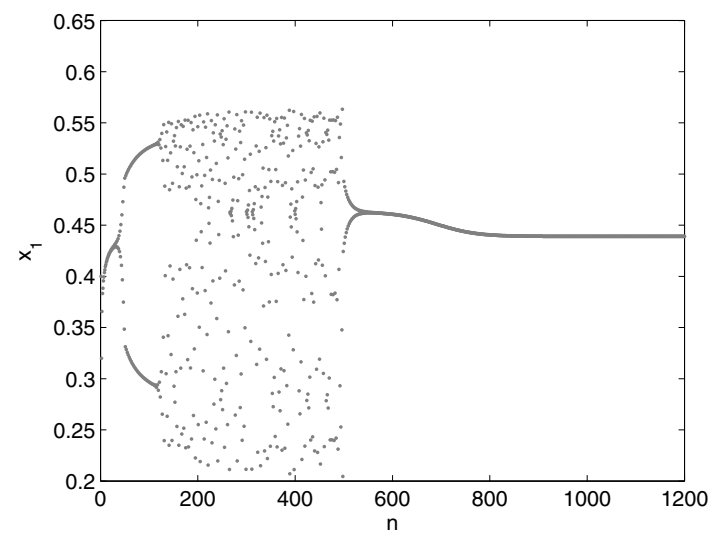

Fig. 5. Time responses for the state $x_{1}$ of the controlled model (78) in the $\left(n, x_{1}\right)$ plane with $\delta=1.78$, and initial values $\left(x_{10}, x_{20}\right)=(0.3,0.1)$. 
1.5184 , the Jacobian matrix $J\left(E_{2}\right)$ has two eigenvalues $\lambda_{1}=-1, \lambda_{2}=0.9716$. Hence, $\left(\delta_{1}, k, \beta, \delta_{0}, \gamma, \delta\right)=$ $(0.8,2.5,0.4,0.6,0.5,1.5184) \in F_{31}$. Further, by computing (30) and (31), we can obtain that $\alpha_{1}=$ $-4.654, \alpha_{2}=43.32$. This illustrates Theorem 1 .

From Fig. 4 we observe that equilibrium $E_{2}$ is stable for $\delta<1.5184$, and there are orbits of period-doubling for $\delta=1.5184$. From $\delta=1.76$ to 1.83 , we can see the attracting chaotic sets, and when $\delta$ from 1.88 to 1.92 , we can see the non-chaotic sets.

We have performed some numerical simulations to see how OGY control stabilizes the unstable periodic orbits embedded in the chaotic attractor. Parameter values are fixed as $\left(\delta_{1}, k, \beta, \delta_{0}, \gamma, \delta\right)=$ $(0.8,2.5,0.4,0.6,0.5,1.5184)$. Figure 5 shows that we do not perform control to the model (2) when $n<500$ (that is, $p \equiv 0$ ), and (2) presents chaotic behavior. When $n>500$, the chaotic orbit falls into a small neighborhood

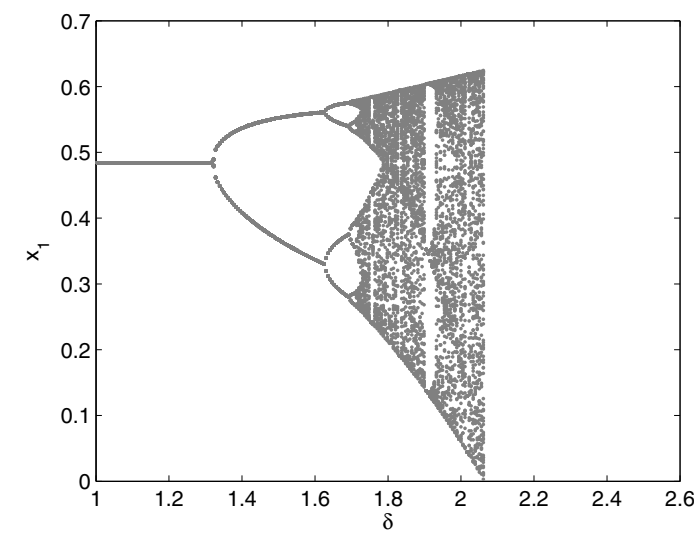

Fig. 6. Flip bifurcation of the model (2) in the $\left(\delta, x_{1}\right)$ plane, and initial values $\left(x_{10}, x_{20}\right)=(0.12,0.0012)$.

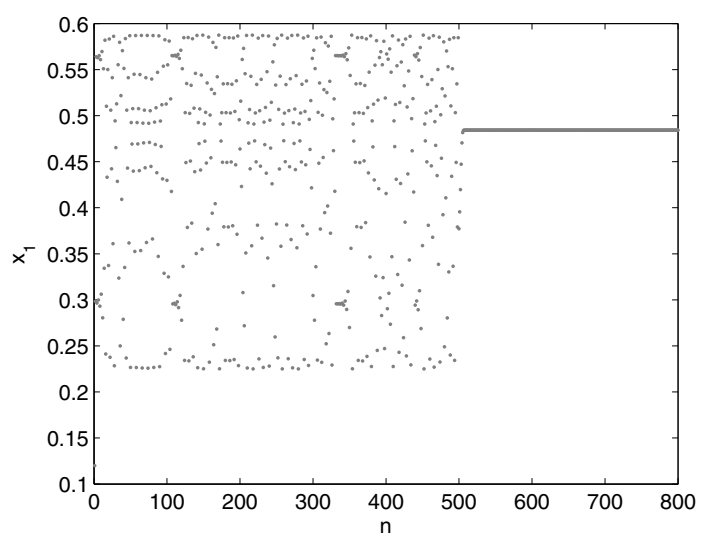

Fig. 7. Time responses for the state $x_{1}$ of the controlled model (78) in the $\left(n, x_{1}\right)$ plane with $\delta=1.78$, and initial values $\left(x_{10}, x_{20}\right)=(0.12,0.0012)$. of the desired equilibrium, we start control, so that the chaotic orbits are quickly stabilized to $E_{2}$.

Case 3. Varying $\delta$ in the range $1 \leq \delta \leq 2.6$, and fixing $\delta_{1}=0.42, k=2.2, \beta=0.273, \delta_{0}=$ $0.71, \gamma=0.43$. By computing, the model (2) has an equilibrium at $E_{1}(0.4842,0)$. When $\delta=1.3123$, the Jacobian matrix $J\left(E_{1}\right)$ has two eigenvalues $\lambda_{1}=$ -1 , and $\lambda_{2}=2.3123$. Hence, $\left(\delta_{1}, k, \beta, \delta_{0}, \gamma, \delta\right)=$ $(0.42,2.2,0.273,0.71,0.43,1.3123) \in F_{A}$. Further, by computing (30) and (31), we can obtain that $\alpha_{1}=$ $-0.1297, \alpha_{2}=3.2875$. This illustrates Theorem 1 .

From Fig. 6 we see that equilibrium $E_{1}$ is stable for $\delta<1.3123$, and loses its stability when $\delta=$ 1.3123. Furthermore, there is flip bifurcation from Fig. 6 . Moreover, a chaotic set is emerged with the increasing of $\delta$, and we know that when $\delta$ increases to 1.7823 the prey tends to extinct, and at last the predator and prey tend to extinction.

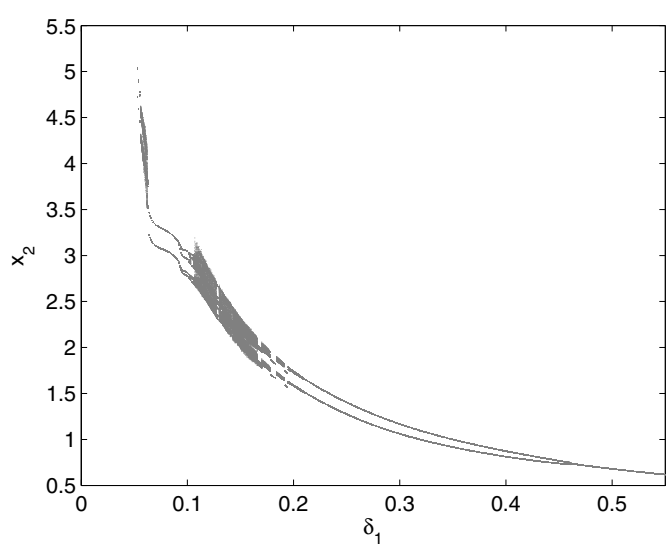

Fig. 8. Saddle-node bifurcation of the model (2) in the $\left(\delta_{1}-x_{2}\right)$ plane, and initial values $\left(x_{10}, x_{20}\right)=(0.12,0.0012)$.

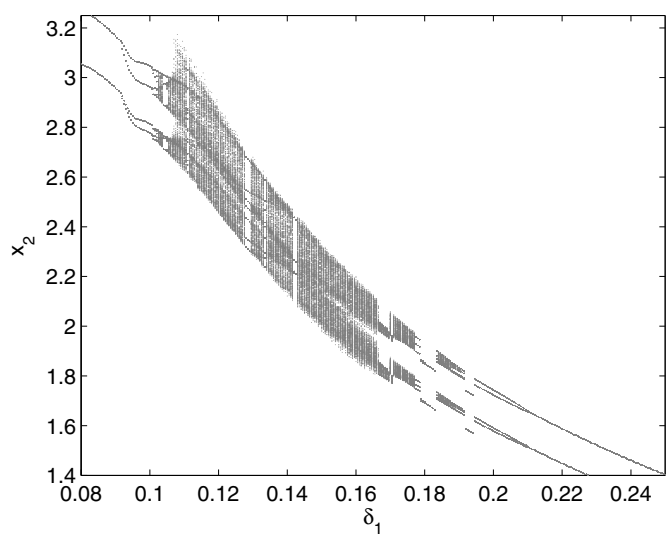

Fig. 9. Local amplification corresponding to Fig. 8 for $\delta_{1} \in$ $[0.08,0.25]$ in the $\left(\delta_{1}, x_{2}\right)$ plane. 
We have performed some numerical simulations to see how the OGY control stabilizes the unstable periodic orbits embedded in the chaotic attractor. Parameter values are fixed as $\left(\delta_{1}, k, \beta, \delta_{0}, \gamma, \delta\right)=$ $(0.42,2.2,0.273,0.71,0.43,1.3123)$. Figure 7 shows that we do not perform control to model (2) when $n<500$ (that is, $p \equiv 0$ ), and model (2) presents chaotic behavior. When $n>500$, the chaotic orbits falls into small neighborhood of the desired equilibrium, we start control, so that the chaotic orbits is quickly stabilized to $E_{1}$.

Case 4. Varying $\delta_{1}$ in the range $0.04 \leq \delta_{1} \leq 0.7$, and fixing $\delta=0.23, k=2.2, \beta=0.273, \delta_{0}=$ $-0.34, \gamma=0.23$. By computing (4), when $\delta_{1}=0.4712$, the model (2) has a positive equilibrium $(0.1375,0.0731)$ and the Jacobian matrix $J\left(E_{2}\right)$ has two eigenvalues $\lambda_{1}=1, \lambda_{2}=-3.9741$. Hence, $\left(\delta_{1}, k, \beta, \delta_{0}, \gamma, \delta\right)=$ $(0.4712,-0.34,2.2,0.273,0.23,0.23) \in F_{34}$. Further, for smaller $\delta_{1}=0.0812$, by computing (4), the model (2) has two positive equilibria $(0.0063,4.4239)$ and (0.0274, 5.2163). By computing (74)-(77), we can obtain that $\eta_{1}=-4.9255, \eta_{2}=21.3847, \eta_{3}=3.2988, \eta_{4}=$ -0.2319 . This illustrates Theorem 3 .

We give the saddle-node bifurcation diagram Figs. 8 and 9 of the model (2) in the $\left(\delta_{1}, x_{2}\right)$ plane for $\delta_{0}=$ $-0.34, k=2.2, \beta=0.273, \gamma=0.23, \delta=0.23$, and $0.04 \leq \delta_{1} \leq 0.7$. From Figs. 8 and 9, we can see that period- 2 occurs at $\delta_{1}=0.0812$, period- 4 occurs at $\delta_{1}=0.1183$, and cascades of period-halving bifurcation and flip bifurcation can be seen. And as $\delta_{1}$ increases from 0.1724 to 0.1863 , the model (2) goes through quasi-periodicity. When $\delta_{1}$ increases to 0.4712 , the model (2) becomes stable. This illustrates Theorem 3.

\section{Discussion}

In this paper, we discuss the dynamical behaviors of the model (2), precisely by choosing the step size $\delta$ and the death rate of the predators $\delta_{1}$ as a bifurcation parameter, it has been shown that the model undergoes a series of bifurcations including a flip bifurcation, a Hopf bifurcation, and a saddle-node bifurcation. We obtain these bifurcations by only changing the bifurcation parameters; we also obtain flip bifurcation and chaos at boundary equilibrium $(m, 0)$ by choosing $\delta$ as a bifurcation parameter. At the same time, as the parameters vary, the model exhibits a stable period-one orbit, a period-two orbit, a period- $n$ orbit or coexistence of several periodic orbits, an invariant cycle, chaotic oscillators, even a sudden onset of chaos suddenly and the chaotic dynamics approach to the period orbits, inverse period-doubling orbits. Furthermore, we obtain that the preys and predators can tend to stable equilibria when the preys and predators are in a chaotic dynamic by using chaos control.
However, for the continuous-time two-species prey-predator model (1), from the article by Liu et al. (2010) we see that the dynamical behaviors are quite simple. In particular, if there exists a unique positive equilibrium $E_{2}\left(x_{1}^{*}, x_{2}^{*}\right)$, then $E_{2}\left(x_{1}^{*}, x_{2}^{*}\right)$ can be global asymptotically stable under some certain conditions, or there exists a unique stable limit cycle around $E_{2}\left(x_{1}^{*}, x_{2}^{*}\right)$. Therefore, from the analysis and results in this paper we see that the discrete-time two-species prey-predator model (2) can produce a much richer set of patterns than those observed in the corresponding continuous-time model (1)

\section{Acknowledgment}

The authors are very grateful to the editor and anonymous referees for their valuable comments and helpful suggestions, which led to a substantial improvement of the original manuscript. This work was supported by the National Natural Science Foundation of China (Grants No. 11271312, 11001235) and the Natural Science Foundation of Xinjiang (Grants No. 2012211B07, 2011211B08).

\section{References}

Busłowicz, M. (2012). Robust stability of positive continuous-time linear systems with delays, International Journal of Applied Mathematics and Computer Science 20(4): 665-670, DOI: 10.2478/v10006-010-0049-8.

Busłowicz, M. and Ruszewski, A. (2012). Computer method for stability analysis of the Roesser type model of 2D continous-discrete linear systems, International Journal of Applied Mathematics and Computer Science 22(2): 401-408, DOI: 10.2478/v10006-012-0030-9.

Duda, J. (2012). A Lyapunov functional for a system with a time-varying delay, International Journal of Applied Mathematics and Computer Science 22(2): 327-337, DOI: 10.2478/v10006-012-0024-7.

Fan, Y. and Li, W. (2004). Permanence for a delayed discrete ratio-dependent predator-prey system with Holling type functional response, Journal of Mathematical Analysis and Applications 299(2): 357-374.

Freedman, H.I. (1976). Graphical stability, enrichment, and pest control by a natural enemy, Mathematical Biosciences 31(3-4): 207-225.

Freedman, H.I. (1980). Deterministic Mathematical Models in Population Ecologys, Marcel Dekker, New York, NY.

Grebogi, C., Ott, E. and Yorke, J.A. (1983). Crises, sudden changes in chaotic attractors, and transient chaos, Physica D: Nonlinear Phenomena 7(1-3): 181-200.

Grebogi, C., Ott, E. and Yorke, J.A. (1986). Critical exponent of chaotic transients in nonlinear dynamical systems, Physical Review Letters 57(11): 1284-1287. 
Grebogi, C., Ott, E., Romeiras, F. and Yorke, J.A. (1987). Critical exponents for crisis-induced intermittency, Physical Review A 36(11): 5365-5380.

Guckenheimer, J. and Holmes, P. (1983). Nonlinear Oscillations, Dynamical Systems, and Bifurcations of Vector Fields, Springer-Verlag, New York, NY.

Hainzl, J. (1988). Stability and Hopf bifurcation in a predator-prey system with several parameters, SIAM Journal on Applied Mathematics 48(1): 170-190.

Harrison, G.W. (1986). Multiple stable equilibria in a predator-prey system, Bulletin of Mathematical Biology 48(2): 137-148.

He, Z. and Lai, X. (2011). Bifurcation and chaotic behavior of a discrete-time predator-prey system, Nonlinear Analysis: Real World Applications 12(1): 403-417.

Holling, C.S. (1965). The functional response of predators to prey density and its role in mimicry and population regulation, Memoirs of the Entomological Society of Canada 97(45): 1-60.

Hsu, S.B. (1978). The application of the Poincare-transform to the Lotka-Volterra model, Journal of Mathematical Biology 6(1): 67-73.

Hu, Z., Teng, Z. and Zhang, L. (2011). Stability and bifurcation analysis of a discrete predator-prey model with nonmonotonic functional response, Nonlinear Analysis: Real World Applications 12(4): 2356-2377.

Huang, J. and Xiao, D. (2004). Analyses of bifurcations and stability in a predator-prey system with Holling type-IV functional response, Acta Mathematicae Applicatae Sinica 20(1): $167-178$.

Jing, Z. (1989). Local and global bifurcations and applications in a predator-prey system with several parameters, Systems Science and Mathematical Sciences 2(4): 337-352.

Jing, Z. , Chang, Y. and Guo, B. (2004). Bifurcation and chaos in discrete FitzHugh-Nagumo system, Chaos, Solitons \& Fractals 21(3): 701-720.

Jing, Z. and Yang, J. (2006). Bifurcation and chaos in discrete-time predator-prey system, Chaos, Solitons \& Fractals 27(1): 259-277.

Kazarinoff, N.D. and Van Den Driessche, P. (1978). A model predator-prey system with functional response, Mathematical Biosciences 39(1-2): 125-134.

Kuznetsov, Y.A. (1998). Elements of Applied Bifurcation Theory, Springer-Verlag, Berlin.

Liu, X. and Xiao, D. (2007). Complex dynamic behaviors of a discrete-time predator-prey system, Chaos, Solitons \& Fractals 32(1): 80-94.

Liu, W., Li, D. and Yao, T. (2010). Qualitative analysis of Holling IV predator-prey system with double density retrie, Natural Sciences Journal of Harbin Normal University 26(6): 8-12, (in Chinese).

Ott, E., Grebogi, C. and Yorke, J.A. (1990). Controlling chaos, Physical Review Letters 64(11): 1196-1199.
Raja, R., Sakthivel, R., Anthoni, S.M. and Kim, H. (2011). Stability of impulsive Hopfield neural networks with Markovian switching and time-varying delays, International Journal of Applied Mathematics and Computer Science 21(1): 127-135, DOI: 10.2478/v10006-011-0009-y.

Robinson, C. (1999). Dynamical Systems, Stability, Symbolic Dynamics and Chaos, 2nd Edn., CRC Press, Boca Raton, FL/London/New York, NY/Washington, DC.

Ruan, S. and Xiao, D. (2001). Global analysis in a predator-prey system with nonmonotonic functional response, SIAM Journal on Applied Mathematics 61(4): 1445-1472.

Scholl, E. and Schuster, H.G. (2008). Handbook of Chaos Control, Wiley-VCH, Weinheim.

Tong, Y., Liu, Z. and Wang, Y. (2012). Existence of period solutions for a predator-prey system with sparse effect and functional response on time scales, Communications in Nonlinear Science and Numerical Simulation 17(8): 3360-3366.

Wang, Y., Jing, Z. and Chan, K. (1999). Multiple limit cycles and global stability in predator-prey model, Acta Mathematicae Applicatae Sinica 15(2): 206-219.

Wiggins, S. (1990). Introduction to Applied Nonlinear Dynamical Systems and Chaos, Springer-Verlag, Berlin.

Wolkowicz, G.S.K. (1988). Bifurcation analysis of a predator-prey system involving group defence, SIAM Journal on Applied Mathematics 48(3): 592-606.

Xu, C., Liao, M. and He, X. (2011). Stability and Hopf bifurcation analysis for a Lotka-Volterra predator-prey model with two delays, International Journal of Applied Mathematics and Computer Science 21(1): 97-107, DOI: 10.2478/v10006-011-0007-0.

Zhang, Q., Yang, L. and Liao, D. (2011). Existence and exponential stability of a periodic solution for fuzzy cellar neural networks with time-varying delays, International Journal of Applied Mathematics and Computer Science 21(4): 649-658, DOI: 10.2478/v10006-011-0051-9.

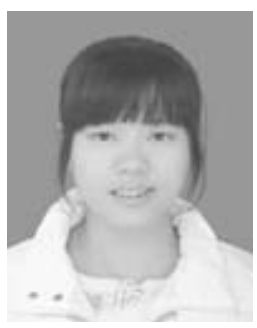

Qiaoling Chen is an M.Sc. student at the College of Mathematics and Systems Science, Xinjiang University, Urumqi, China. She received the B.Sc. degree from Xinjiang University in 2010. Her research interests focus on dynamical behaviors of differential and difference equations, including bifurcation theory and chaotic control.

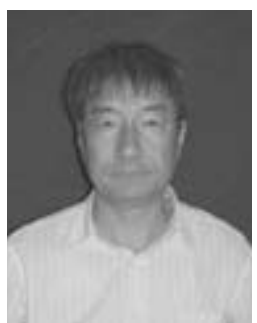

Zhidong Teng is a professor at the College of Mathematics and Systems Science, Xinjiang University, Urumqi, China. He received the Ph.D. from Al-Farabi Kazakh National University, Alma-Ata, in 1995. His research interests focus on the theory of differential and difference equations, population dynamical systems, epidemic models, and neural network systems. 


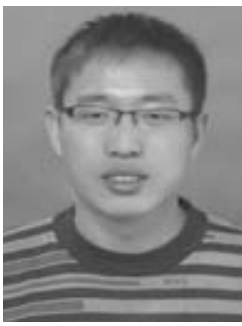

Zengyun Hu is a Ph.D. student at the College of Mathematics and Systems Science, Xinjiang University, Urumqi, China, and an assistant research fellow at the Xinjiang Institute of Ecology and Geography, Chinese Academy of Sciences. He received his M.Sc. degree from Xinjiang University in 2011. His research interests focus on discrete systems and epidemic models.

Received: 13 April 2012

Revised: 8 September 2012 